\title{
Spinal Capillary Hemangioma: A Rare Benign Extradural Tumor
}

\author{
Naomi Niznick(1), Thanh Binh Nguyen, Pierre R. Bourque
}

Keywords: Spinal neoplasm, Epidural tumors, Neuroimaging

doi:10.1017/cjn.2020.60

Can J Neurol Sci. 2020; 47: 549-550

We report the case of a 51-year-old woman who presented to hospital with progressive lower body sensory symptoms and gait difficulties. Seven months previously, she developed numbness and paresthesia which ascended progressively from her feet to her lower torso. Two months prior to admission, she also developed bilateral leg weakness and gradually noted symptoms of rectal urgency, urinary hesitancy with retention, and saddle anesthesia. On examination, there was mildly asymmetric spastic paraparesis in a pyramidal distribution (MRC 4/5 for hip flexion, hip, and knee flexion) and a T5 sensory level to pinprick. There was bilateral sustained ankle clonus and Babinski signs.

Spinal magnetic resonance imaging (MRI) showed a posterior epidural mass with longitudinal extension from T5 to T6 and lateral extension to the T5-T6 neural foramina. The mass was hyperintense on T2-weighted images and demonstrated strong and homogenous gadolinium enhancement, with the presence of some linear signal flow voids. It caused severe compression of the spinal cord which showed T2 hyperintensity (Figure 1). The patient underwent a T3-T6 laminectomy with uncomplicated complete removal of the extradural tumor. Intraoperatively, the lesion was described as a purple, soft, well-circumscribed vascular tumor directly apposed to the dura. Histological examination demonstrated abundant blood vessels with occasional foamy-looking cells staining positively for the vascular markers CD34 and CD31. There was a low proliferation index, no necrosis, and no positivity for markers of hemangioblastoma or renal cell carcinoma. This pathology was diagnostic of capillary hemangioma. Postoperatively, the patient showed rapid resolution of sphincter dysfunction and there was marked improvement of sensory loss and gait at 3 months.

Hemangiomas are congenital vascular malformations, classified according to the predominant vascular morphology: cavernous, capillary, arteriovenous, or venous. ${ }^{1,2}$ Capillary hemangiomas are the most common subtype overall as they occur frequently in skin and oral mucosa of infants and children. Spinal capillary epidural hemangiomas on the other hand are exceedingly rare, with fewer than 20 reported cases in the literature. ${ }^{1,3-8}$ They are benign tumors, described at all spinal levels, with a marked preponderance at the thoracic level. ${ }^{8}$ The classical clinical presentation is one of spinal pain, radiculopathy, or subacute to chronic myelopathy. The acute syndrome of intratumoral hematoma is distinctly uncommon with capillary hemangiomas, in contrast to the cavernous subtype. ${ }^{1,8,9}$

MRI spine with gadolinium is the imaging modality of choice. It typically demonstrates an iso- or hypointense lesion on T1weighted images, hyperintensity on $\mathrm{T} 2$-weighted images, and homogenous enhancement following gadolinium administration. ${ }^{2}$ These imaging features are not specific, and the differential diagnosis of an enhancing epidural soft tissue mass should include plasmacytoma, lymphoma, and other tumors such as hemangioblastoma, hemangioendothelioma hemangiopericytoma, meningioma, schwannoma, and neurofibroma, which, though typically intradural, can occur in a predominant extradural location. ${ }^{1,2,5,8}$ Surgical en bloc resection is the treatment of choice, with a very favorable functional prognosis if performed early. ${ }^{4}$

Spinal epidural capillary hemangioma should be considered in patients presenting with thoracic myelopathy and MRI findings of a strongly enhancing circumscribed extradural mass with flow voids. With prompt recognition and immediate surgical referral, these benign tumors have a very favorable prognosis.

\section{CONFLict OF INTEREST}

The authors have no conflicts of interest to declare.

\section{Statement of Authorship}

All authors contributed in a meaningful way to the production of this case report.

$\mathrm{NN}$ contributed to the script, figure formatting, editing, and submission of the manuscript.

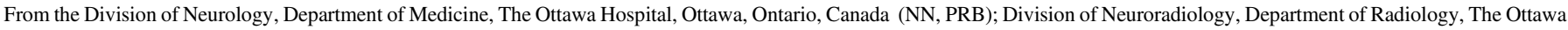
Hospital, Ottawa, Ontario, Canada (TBN) and Ottawa Hospital Research Institute, The Ottawa Hospital, Ottawa, Ontario, Canada (PRB)

Received March 14, 2020. Date of Acceptance March 19, 2020.

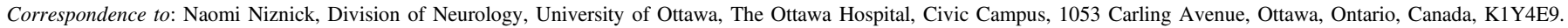
Email: naniznick@toh.ca 
T1

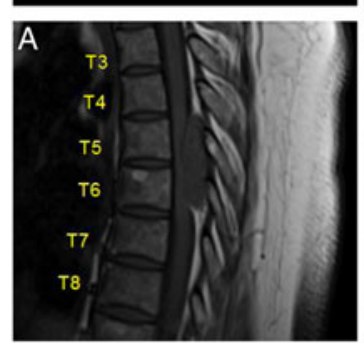

T2
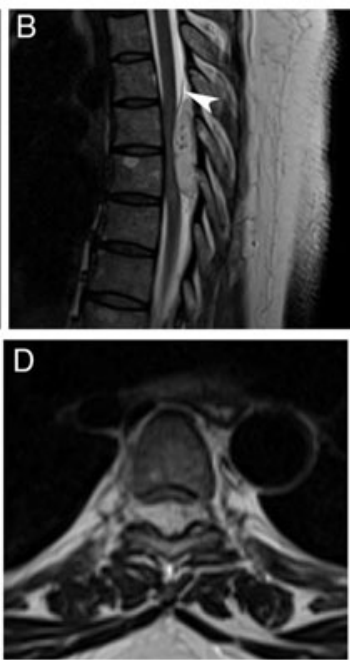

Contrast
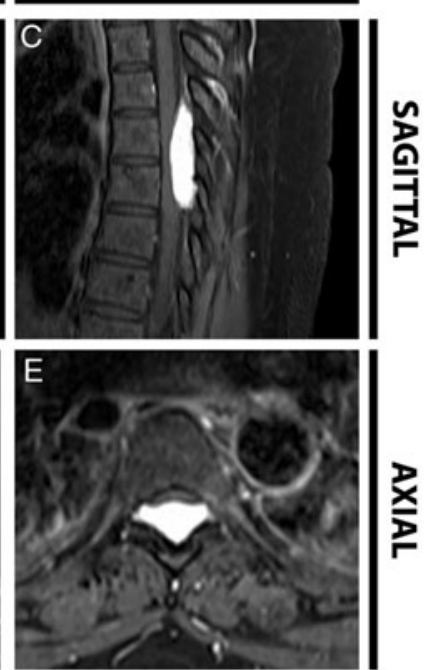

Figure 1: Spine MRI. Sagittal T1 (A) shows an isointense epidural mass located posteriorly at T5-T6. Sagittal T2 (B) shows a hyperintense lesion with intralesional flow voids and the dural margin delineated anteriorly to the tumor (white arrow). Axial T2 (D) demonstrates lateral extension into the T5-T6 neural foramina. There is severe spinal cord compression with associated intramedullary T2 hyperintensity. Post-contrast sagittal (C) and axial T1 (E) demonstrate strong and homogeneous contrast enhancement.

PRB contributed to the script, editing, and submission of the manuscript.

TBN contributed to the imaging interpretation and editing of the manuscript.

\section{REFERENCES}

1. Brasil AVB, Rohrmoser RG, Gago G, Cambruzzi E. Atypical spinal epidural capillary hemangioma: case report. Surg Neurol Int. 2018;9:198.

2. Muhmer M, Bostelmann R, Sarikaya-Seiwert S, Schneiderhan M, Steiger HJ, Cornelius JF. Clinical and radiological presentation of spinal epidural haemangiomas: clinical series in a tertiary care centre during a 10-year period. Eur Spine J. 2014;23(2):404-10.

3. Bozkus H, Tanriverdi T, Kizilkilic O, Tureci E, Oz B, Hanci M. Capillary haemangiomas of the spinal cord: report of two cases. Minim Invasive Neurosurg. 2003;46(1):41-6.
4. Cofano F, Marengo N, Pecoraro F, Penner F, Bertero L, Zenga F. Spinal epidural capillary hemangioma: case report and review of the literature. Br J Neurosurg. 2019:1-4.

5. Rajeev MP, Waykule PY, Pavitharan VM, Nandeesh BN. Spinal epidural capillary hemangioma: a rare case report with a review of literature. Surg Neurol Int. 2017;8:123.

6. Seferi A, Alimehmeti R, Vyshka G, Bushati T, Petrela M. Case study of a spinal epidural capillary hemangioma: a 4-year postoperative follow-up. Global Spine J. 2014;4(1):55-8.

7. Vassal F, Peoc'h M, Nuti C. Epidural capillary hemangioma of the thoracic spine with proximal nerve root involvement and extraforaminal extension. Acta Neurochir (Wien). 2011;153(11): 2279-81.

8. Xu H, Tong M, Liu J, Zhou G, Chen F. Purely spinal epidural capillary hemangiomas. J Craniofac Surg. 2018;29(3):769-71.

9. Esene IN, Ashour AM, Marvin E, et al. Pure spinal epidural cavernous hemangioma: a case series of seven cases. J Craniovertebr Junction Spine. 2016;7(3):176-83. 